\title{
ANÁLISIS TAFONÓMICO DE MICROMAMÍFEROS DEL PLEISTOCENO SUPERIOR DEL QUEQUÉN SALADO, PROVINCIA DE BUENOS AIRES, ARGENTINA
}

\author{
CLAUDIA I. MONTALVO \\ Facultad de Ciencias Exactas y Naturales, UNLPam, Uruguay 151, 6300, Santa Rosa, La Pampa, Argentina. \\ cmontalvo@exactas.unlpam.edu.ar \\ RODRIGO L. TOMASSINI \\ Departamento de Geología Universidad Nacional del Sur, CONICET, San Juan 670, 8000, Bahía Blanca, \\ Buenos Aires, Argentina.rodrigo.tomassini@yahoo.com.ar \\ GRACIELA VISCONTI \\ Facultad de Ciencias Exactas y Naturales, UNLPam, Uruguay 151, 6300, Santa Rosa, La Pampa, Argentina. \\ gvisconti@exactas.unlpam.edu.ar \\ SERGIO I. TIRANTI \\ AUSMA, Universidad Nacional del Comahue, Pasaje de la Paz 235, 8370 San Martín de los Andes, Neuquén, Argentina. \\ sitiranti@hotmail.com
}

\begin{abstract}
TAPHONOMIC ANALYSIS OF THE LATE PLEISTOCENE SMALL MAMMALS OF QUEQUÉN SALADO RIVER, BUENOS AIRES, ARGENTINA. A taphonomic analysis was made on small mammal remains recovered from fossil bone accumulations, interpreted as whole and fragmented pellets, from Upper Pleistocene levels of the Quequén Salado River cliffs (Buenos Aires, Argentina). Additionally, the probable source of the accumulation and some features associated with post-burial preservation processes are discussed. From the available information it is possible, through analyzing the anatomical representation of the remains and the degree of digestion, to show affinities with extant accumulations produced by nocturnal birds of prey. Nevertheless, breakage patterns are biased in this association as it shows much higher values than in a recent analysis based on pellets of extant nocturnal raptors. Diagenetic bone fractures found in skeletal elements have probably hidden the fractures produced at the moment of predation. Microstructural characteristics of remains preserved in the pellets are indicative of good preservation indicating rapid burial. Associated remains, interpreted as originating from disassembled pellets, also may have been buried rapidly as they do not show any weathering. From a biostratigraphic point of view and based on the associated mammalian megafauna, this accumulation can be assigned to the Lujanian Stage/Age of the late Pleistocene.
\end{abstract}

Key words: taphonomy, small mammals, Pleistocene.

RESUMO - A análise tafonômica foi realizada em pequenos restos de mamíferos recuperados de acumulações fossilíferas, interpretadas como pelotas de regurgitação inteiras e fragmentadas, das falésias pleistocênicas do Rio Quequén Salado (Buenos Aires, Argentina). Além disso, a provável fonte da acumulação e algumas características associadas aos processos de pós-soterramento da preservação são discutidas. A partir das informações disponíveis, é possível, através da análise da representação anatômica dos restos e do grau de digestão, mostrar afinidades com acumulações comuns formadas por aves noturnas de rapina. No entanto, os padrões de quebra são tendenciosos para esta associação, pois mostra valores muito mais elevados do que em recente análise feita sobre estas aves. Fraturas ósseas de origem diagenética encontradas em elementos esqueletais provavelmente ocultam as fraturas produzidas no momento da predação. Microestruturas características dos restos conservados em pelotas são indicativos de boa preservação, indicando um soterramento rápido. Elementos associados, interpretados como provenientes de pelotas desagregadas também podem ter sido soterrados rapidamente, não apresentando intemperismo. Do ponto de vista bioestratigráfico e com base nos megamamíferos associados, essa assembleia fossilífera pode ser atribuída ao Piso/Idade Lujanense, Pleistoceno tardio.

Palavras-chave: tafonomia, pequenos mamíferos, Pleistoceno.

\section{INTRODUCCIÓN}

En niveles pleistocénicos de la Región Pampeana de Argentina es frecuente el hallazgo de concentraciones de restos fósiles de pequeños vertebrados, principalmente roedores. Sin embargo, es habitual que los trabajos en ese sentido se ocupen principalmente del análisis de los taxones presentes, resultando poco frecuentes las evaluaciones tafonómicas que ayuden a interpretar el origen de estas concentraciones (Pardiñas, 2001, 2004; Ortiz \& Pardiñas, 2001; Cenizo \& de los Reyes, 2008). Uno de los procesos planteados para explicar el origen de estas acumulaciones 
de microvertebrados es la actividad depredadora, tanto por parte de aves rapaces nocturnas y diurnas, como de mamíferos carnívoros (e.g. Pardiñas, 1999; Gómez, 2005, 2007).

Andrews (1990) estableció una metodología sistemática que plantea el reconocimiento del posible depredador involucrado, considerando las alteraciones que éstos producen en los huesos de sus presas. Así, definió tres grupos de depredadores que ocasionan diferentes tipos de modificaciones. Las aves rapaces nocturnas son las que provocan el menor grado de modificación, en tanto en general, los mamíferos carnívoros causan las modificaciones más fuertes (al respecto ver Montalvo et al., 2007; Bennàsar Serra, 2010). Las aves rapaces diurnas provocan modificaciones intermedias entre los otros dos grupos. Dicha distinción se fundamenta en el análisis de la representación anatómica de los diferentes elementos esqueléticos preservados y los grados de rotura y digestión que éstos presentan. Esta metodología fue utilizada por numerosos autores (e.g. Gómez, 2005, 2007; Gómez \& Kaufmann, 2007; Montalvo et al., 2007, 2008, 2012a; Montalvo \& Tallade, 2009, 2010; Montalvo \& Tejerina, 2009) para evaluar las modificaciones que distintos depredadores de la Región Pampeana producen sobre los huesos de pequeños mamíferos presa, en particular roedores.

Estos análisis actualísticos son de utilidad para interpretar qué tipo de depredador pudo haber participado en la producción de acumulaciones fósiles (Bennàsar Serra, 2010). Por otro lado, al determinar que la acumulación fue producida por un depredador, se puede ajustar el rango temporal de su formación, limitándolo a lapsos cortos (Behrensmeyer \& Hook, 1992). Por lo expuesto, esas asociaciones son también importantes desde el punto de vista biocronológico y bioestratigrafico (Verzi et al., 2008).

Se presenta aquí el análisis tafonómico realizado sobre restos de micromamíferos recuperados de acumulaciones fósiles procedentes de egagrópilas enteras y desagregadas, recuperadas de un depósito pleistocénico interpretado como una llanura de inundación en las barrancas del río Quequén Salado, Buenos Aires, Argentina. Los datos obtenidos fueron comparados con resultados de evaluaciones de acumulaciones producidas por depredadores actuales de la Región Pampeana. Se discute el posible productor de la asociación fósil y la asignación temporal de la misma. Además se evalúan algunos caracteres vinculados con su preservación posterior al enterramiento.

\section{CONTEXTO GEOGRÁFICO Y ESTRATIGRÁFICO}

Las primeras menciones y descripciones sobre niveles cenozoicos en el río Quequén Salado corresponden a los estudios realizados por Frenguelli (1928). Posteriormente, diversos autores (e.g. Alberdi et al., 1989; Pardiñas et al., 1996; Schillizzi et al., 2006) hicieron referencia a la existencia de depósitos correspondientes al Pleistoceno Superior a lo largo del curso de este río, los cuales, según sus características sedimentarias y la fauna de mamíferos fósiles presentes, en la mayoría de los casos fueron correlacionados con la Formación Luján (Fidalgo et al., 1973) y sus distintos miembros.

La localidad de la que provienen los materiales evaluados $\left(38^{\circ} 26^{\prime} 14,14\right.$ 'S, $\left.60^{\circ} 40^{\prime} 13,29^{\prime \prime} \mathrm{O}\right)$, se encuentra ubicada aproximadamente a $15 \mathrm{~km}$ al noreste de la localidad de Irene (partido de Coronel Dorrego, Provincia de Buenos Aires) y a unos $175 \mathrm{~km}$ al noreste de la ciudad de Bahía Blanca (Figura 1). La secuencia sedimentaria, ubicada sobre la margen este del río, aflora en una gran extensión, con alturas variables entre 15 y 20 m (Figura 2).

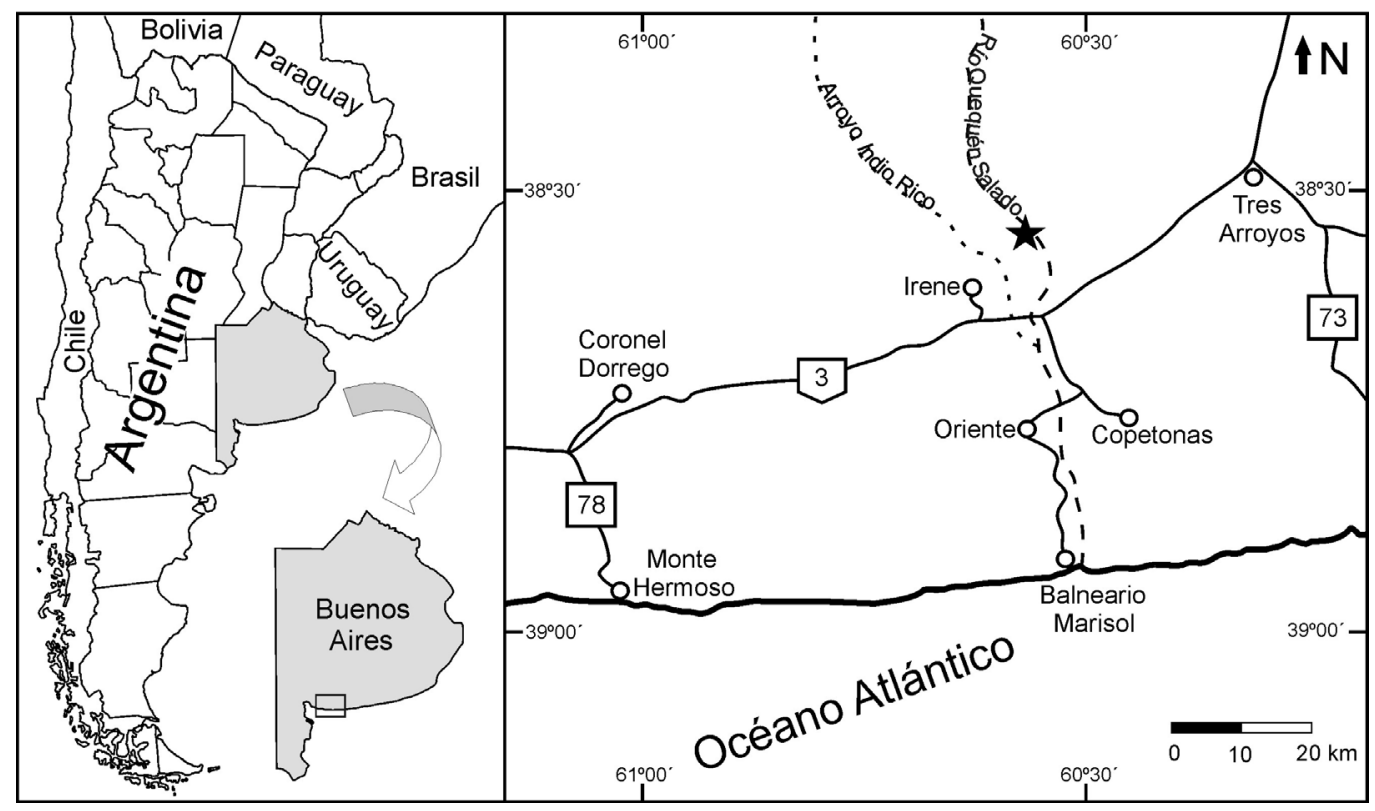

Figura 1. Mapa de ubicación del yacimiento fosilífero en el río Quequén Salado, Provincia de Buenos Aires, Argentina. La estrella muestra la zona de la que proviene la asociación estudiada.

Figure 1. Location map of fossiliferous site in Quequén Salado River, Buenos Aires Province, Argentina. The star shows the area from which comes the assemblage studied. 


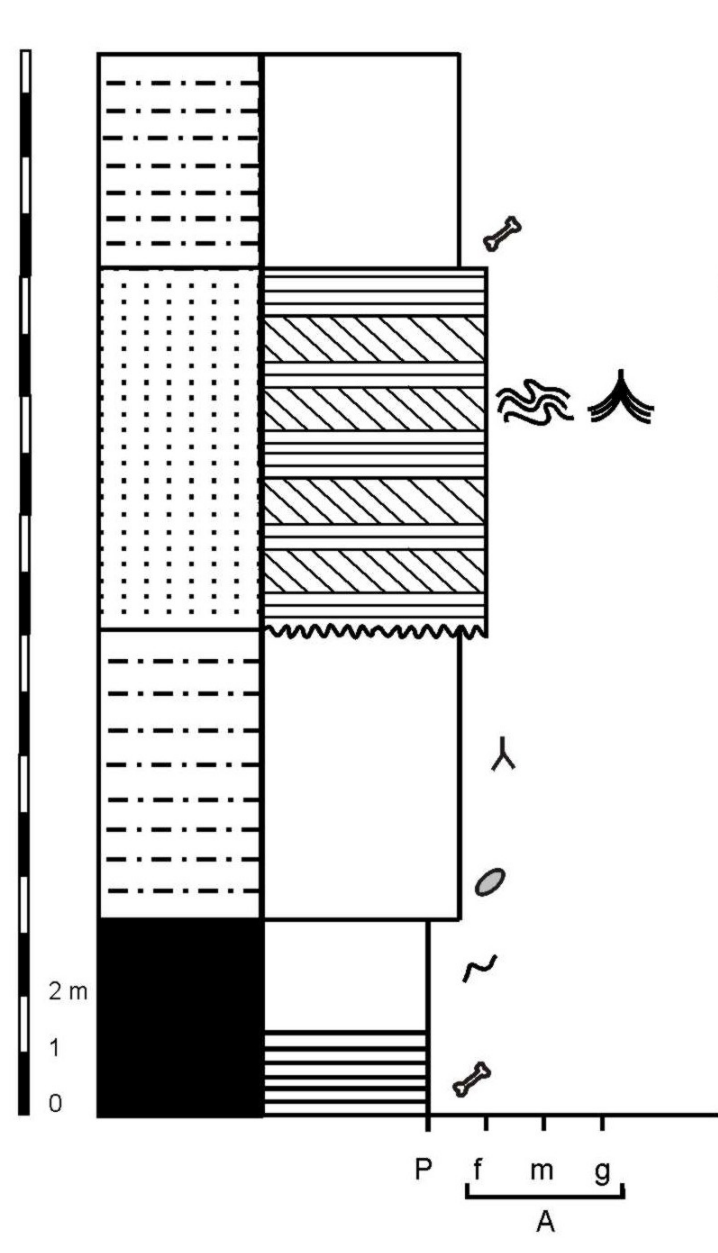

\section{Litología}

\begin{tabular}{|ll}
\hline & Limolitas arcillosas \\
\hline$:=::$ Limolitas \\
\hline$\vdots$ : Areniscas
\end{tabular}

\section{Superficies}

mman Erosiva

Neta

\section{Estructuras sedimentarias}

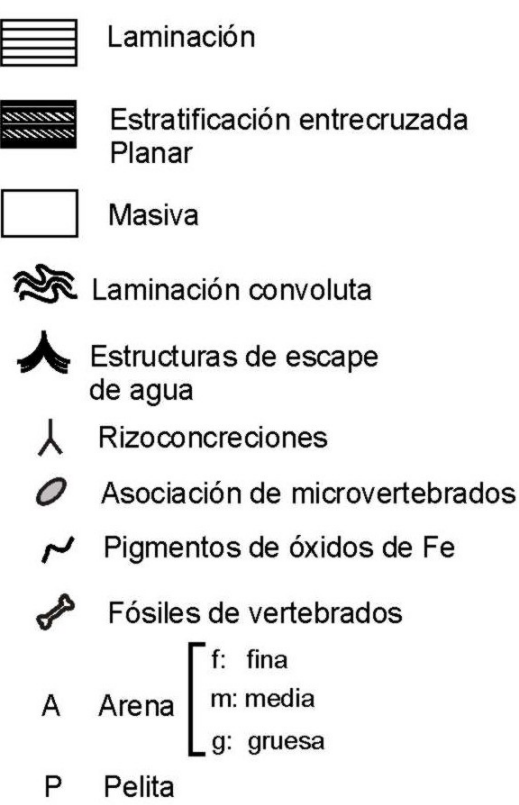

Figura 2. Perfil estratigráfico del área del Quequén Salado portadora de la asociación estudiada. Se interpreta como depósitos de llanura de inundación sobre el que se labró un canal fluvial.

Figure 2. Stratigraphic section of the Quequén Salado area of provenance of assemblage studied. It is interpreted as floodplain deposits on which a fluvial channel was worked.

La sucesión estratigráfica, con base cubierta por el nivel actual del río, comienza con 3,5 $\mathrm{m}$ de limolitas arcillosas pardo oscuras (5 YR 2/2) a pardo rojizas (10 R 4/6), finamente laminadas en el sector basal y macizas desde el sector medio hacia el techo, con abundantes pátinas de óxidos de hierro. Superpuesto, en forma transicional, se reconocieron $4,5 \mathrm{~m}$ de limolitas pardo claras (5 YR 5/6) a pardo rojizo claras (10 R 5/4), macizas, poco consolidadas y friables. Éstas constituyen un nivel horizontal tabular, de gran extensión lateral y espesor constante, en el que se observan numerosas rizoconcreciones. Cuarzo, plagioclasas, trizas de vidrio volcánico y óxidos de hierro y de manganeso se reconocieron como los principales componentes minerales en todo este nivel. La asociación de micromamíferos estudiada proviene de la base de este estrato, y se encontraba distribuida en un espesor de escasos centímetros (Figura 2).

Esta secuencia representaría un ambiente de llanura de inundación, originado a partir de la decantación de los materiales finos aportados por un canal fluvial durante sucesivos eventos de inundación. La presencia de rizoconcreciones permitiría interpretar el desarrollo incipiente de paleosuelos sobre los depósitos mencionados.
Por encima de estos niveles de llanura de inundación, dispuestos sobre un contacto erosivo, se identificaron $6 \mathrm{~m}$ de areniscas finas parcialmente consolidadas, de color pardo claro (5 YR 6/4) a pardo amarillento pálido (10 YR 6/2), con estratificación entrecruzada planar. En algunos sectores se observaron estructuras correspondientes a escapes de agua y otras deformacionales gravitativas, como laminación convoluta. En contacto neto, se encuentra un nivel suprayacente comprendido por $3,5 \mathrm{~m}$ de limolitas pardo amarillentas, muy consolidadas y macizas. Mineralógicamente se reconocieron los mismos materiales presentes en los depósitos de llanura de inundación.

Teniendo en cuenta que la estratificación entrecruzada planar se produce debido a la migración de megaóndulas de cresta recta, por la acción de una corriente unidireccional, se interpretó que estos depósitos corresponderían al relleno de un canal fluvial, labrado sobre los sedimentos de la llanura de inundación. Por otro lado, el desarrollo de laminación convoluta indicaría la licuefacción y deformación, por presión litostática, de los sedimentos saturados en agua previamente depositados en el interior del canal.

De los niveles inferior y superior de esta sucesión 
estratigráfica, durante las campañas de prospección, fueron recuperados además de los materiales que aquí se analizan, restos fósiles de megamamíferos, asignados a Macrauchenia patachonica Owen, 1838 (Litopterna) y a Glyptodon reticulatus Owen, 1845 (Xenarthra).

\section{MATERIAL Y MÉTODOS}

El material analizado en este trabajo, que consiste en restos fósiles aislados de microvertebrados y cuatro acumulaciones en las que los fósiles se encontraban concentrados, están depositados en la colección del Museo Municipal de Ciencias Naturales "Vicente Di Martino" de la localidad de Monte Hermoso, Provincia de Buenos Aires, Argentina, bajo el acrónimo MMHQEQ-09-11-01. Las cuatro muestras con fósiles acumulados no fueron desagregadas, y sólo una de ellas se utilizó para realizar cortes delgados. Los materiales aislados de microvertebrados estaban concentrados en un área reducida y fueron recuperados mediante tamizado del sedimento del nivel de las acumulaciones. Como se indicó, todos los materiales estudiados provienen del sector inferior de los depósitos de llanura de inundación y fueron exhumados de un espesor comprendido entre 5 y $6 \mathrm{~cm}$.

La determinación de los colores descriptos para las sedimentitas se realizó utilizando la Rock Color Chart (Goddard et al., 1948).

En laboratorio, se analizaron tafonómicamente los restos fósiles asignados a roedores a fin de determinar las evidencias que permitieran identificar los patrones de modificación del posible productor de las acumulaciones. Las identificaciones taxonómicas fueron realizadas por uno de los autores (SIT) utilizando para ello materiales de su propia colección, bibliográficos y ejemplares de la colección paleontológica de la Facultad de Ciencias Exactas y Naturales (UNLPam). A fin de analizar el tamaño de los individuos que formaron parte de esta asociación, se consideraron las masas corporales estimadas para los taxones presentes evaluadas por Tiranti (1992).

Para la muestra completa fueron calculados los siguientes índices: NEIT (número de especímenes identificados por taxón), NMI (número mínimo de individuos) y NME (número mínimo de elementos esqueléticos). En este último índice no se consideraron los 152 fragmentos craneanos (huesos planos y otras porciones de la base del cráneo) y entre los metapodios se incluyeron los 30 astrágalos y 38 calcáneos identificados. Se utilizó la metodología tafonómica propuesta por Andrews (1990) y Fernández-Jalvo \& Andrews (1992) que incluye: (i) cálculo de la abundancia relativa de los diferentes elementos esqueléticos colectados, considerando la representatividad de cada uno de ellos en el contexto del número mínimo de individuos NMEi/(EixNMI) x 100, donde NMEi es el número mínimo de elementos esqueléticos recuperados en la muestra y Ei es el número esperado de cada elemento en el esqueleto de las presas; (ii) cálculo de índices que evalúan la proporción de los diferentes elementos esqueléticos recuperados: (fémures + húmeros) / (mandíbulas + maxilares); (fémures + tibias + húmeros + radios + ulnas) $\times 8 /$ (mandíbulas + maxilares + molares) x 5; (tibias + radios) / (fémures + húmeros); y finalmente la proporción relativa de molares e incisivos aislados calculados en relación con el número de alvéolos vacíos en mandíbulas y maxilares; (iii) evaluación del grado de rotura de los elementos esqueléticos (húmeros, fémures, ulnas y tibias), considerando por un lado los enteros y por otro los rotos (epífisis proximales y distales y diáfisis); y (iv) análisis del grado de corrosión digestiva, evaluando principalmente las modificaciones producidas en las porciones proximales de fémures, las distales de húmeros, molares e incisivos. Para evaluar la digestión sobre los restos dentarios se tuvo en cuenta si éstos estaban insertos en los alvéolos o aislados.

Los restos fósiles fueron estudiados mediante un microscopio binocular Leica Ms5, y algunos ejemplares seleccionados se fotografiaron con un microscopio electrónico de barrido Jeol 35 CF SEM a $8 \mathrm{kV}$ de la Unidad de Administración Territorial, UAT, CONICET Bahía Blanca (CCT CONICET - BB), Bahía Blanca, Argentina.

La composición química elemental de los elementos esqueléticos, matriz portadora y roca hospedante de los fósiles, se determinó mediante un sistema EDX (Energy Dispersive X-ray), marca EDAX, modelo DX-4, con capacidad de detección desde Boro hasta Uranio. Las características mineralógicas y el grado de preservación de la estructura histológica original se evaluaron sobre dos secciones delgadas, realizadas en una de las acumulaciones, con un microscopio petrográfico Nikon Eclipse E400-POL. Las secciones delgadas se realizaron en el Laboratorio de Petrotomía del INGEOSUR (CONICET), Departamento de Geología (UNS) siguiendo la metodología indicada por Tomassini (2012).

\section{RESULTADOS}

La asociación estudiada, recuperada de los depósitos de llanura de inundación, consiste en numerosos restos aislados de microvertebrados, principalmente roedores. Se recuperaron además cuatro acumulaciones fosilíferas concentradas en una matriz muy consolidada que formaba estructuras ovoides cuyas medidas máximas aproximadas son $4 \mathrm{~cm}$ de largo y $2 \mathrm{~cm}$ de ancho (Figura 3). Los restos aislados y las acumulaciones fosilíferas asociadas, se consideraron como parte de la misma asociación por su proximidad espacial y su coherencia taxonómica y tafonómica.

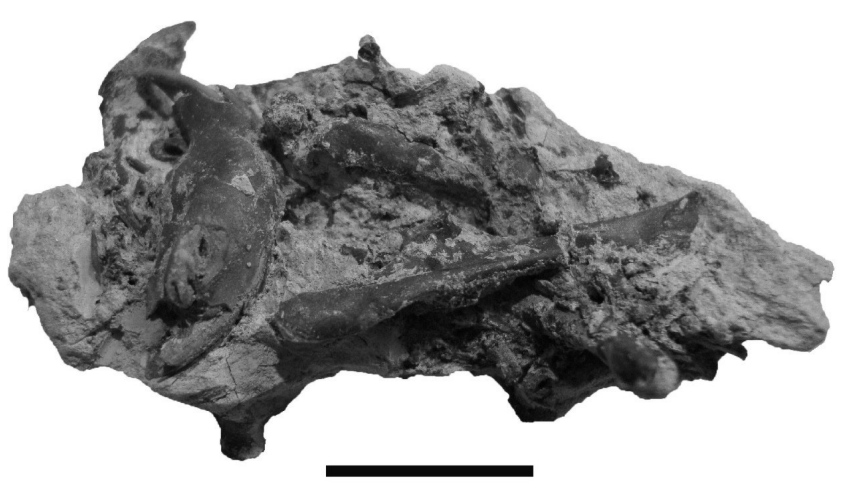

Figura 3. Egagrópila entera. Se observa una hemimandíbula de Ctenomys sp. y varios huesos largos. Escala $=10 \mathrm{~mm}$.

Figure 3. Whole pellet. There is a jaw of Ctenomys sp. and several long bones. Scale bar $=10 \mathrm{~mm}$. 
Desde el punto de vista taxonómico la asociación está constituida por el marsupial Lestodelphys halli (Thomas, 1921) (Didelphimorphia, Didelphidae), los roedores sigmodontinos Reithrodon auritus (Fischer, 1814) y Eligmodontia sp. (Cricetidae) y el caviomorfo Ctenomys sp. (Ctenomyidae). Además, dos elementos esqueléticos se asignaron a reptiles Iguanidae. Las masas corporales estimadas para los taxones presentes varían entre 17 y $250 \mathrm{~g}$ (Tiranti, 1992).

Se recuperaron 3.119 restos fósiles de roedores. De éstos, el $26,58 \%$ eran pequeños fragmentos o esquirlas indeterminables que no se consideraron en el análisis tafonómico. El NEIT obtenido fue de 2.290, mientras que el NME fue de 1.795. El NMI, evaluado sobre la base de las mandíbulas, fue de 34. El promedio de abundancia relativa de los diferentes elementos esqueléticos fue de 54,81\% (Tabla 1).

Tabla 1. Número mínimo de elementos (NME) recuperados de la asociación del Quequén Salado y su abundancia relativa.

Table 1. Minimal number of elements (NME) recovered from Quequén Salado assemblage and their relative abundance.

\begin{tabular}{ccc}
\hline & NME & \% Abundancia relativa \\
\hline mandíbulas & 65 & 95,59 \\
maxilares & 49 & 72,06 \\
escápulas & 23 & 33,82 \\
húmeros & 64 & 94,12 \\
radios & 34 & 50,00 \\
ulnas & 42 & 61,76 \\
pelvis & 43 & 63,24 \\
fémures & 62 & 91,18 \\
tibias & 49 & 72,06 \\
vértebras & 313 & 25,57 \\
incisivos & 56 & 41,18 \\
molares & 86 & 21,08 \\
metapodios & 791 & 41,54 \\
costillas & 118 & 4,17 \\
Total & 1.795 & \\
Promedio & & 54,81 \\
\hline
\end{tabular}

La Tabla 2 muestra los resultados de los índices calculados para evaluar la representatividad de los elementos craneanos y postcraneanos, así como la frecuencia de dientes aislados.

Tabla 2. Índices evaluados en la asociación del Quequén Salado.

Table 2. Indexes assessed from Quequén Salado assemblage.

\begin{tabular}{lc}
\hline (fémur + tibia + húmero + radio + ulna) x 8 / (mandíbula + & 199,8 \\
maxilar + molar) x 5 & 109,6 \\
(fémur + húmero) / (mandíbula + maxilar) & 65,9 \\
(tibia + radio) / (fémur + húmero) & 103,61 \\
\% molares aislados & 311,11 \\
\hline incisivos aislados
\end{tabular}

Entre los restos asignados a Ctenomys sp., el roedor de mayor tamaño en la asociación, los cráneos completos resultaron escasos. Sólo uno de ellos retuvo todos los dientes y, por lo general, tanto la base craneana como la región occipital no se preservaron. Los cráneos de sigmodontinos mostraron un grado de rotura aún mayor. Considerando los restos craneanos de todos los taxones identificados, se determinó que el $87,75 \%$ correspondían a porciones de hemimaxilares. De estos últimos, el $60 \%$ sólo retenía uno o dos molares. Por otro lado, se destaca que en estos restos craneanos, las fracturas de borde liso resultaron muy frecuentes.

Las mandíbulas de Ctenomys sp. fueron las más completas. En toda la asociación, más del $44 \%$ de las mismas presentó la porción articular rota. Además, el 33,80\% carecía del incisivo y sólo el 15,38\% retenía todos los dientes. El 17,44\% de los molares aislados recuperados estaban rotos, en tanto que todos los que se mantuvieron in situ estaban enteros.

Entre los elementos postcraneanos considerados para analizar el grado de rotura, sólo el $23,72 \%$ estaban enteros (Tabla 3). Por lo general las porciones proximales fueron las más frecuentes $(37,15 \%)$, excepto para los húmeros, entre los que predominaron las porciones distales. Sobre el total de fémures, húmeros, tibias y ulnas rotos, el 11,55\% presentaba fracturas transversales de borde liso, en tanto en el resto se identificaron fracturas espirales o escalonadas (Marshall, 1989). Otros elementos, como los metapodios, estaban principalmente enteros ( $84,23 \%$ del total). Entre las escápulas sólo se preservaron las porciones articulares y no se recobró ninguna pelvis entera.

Tabla 3. Grado de rotura en elementos postcraneanos de la asociación del Quequén Salado.

Table 3. Degree of breakage of postcranial elements from Quequén Salado assemblage.

\begin{tabular}{ccccc}
\hline & \% fémur & \% húmero & \% tibia & \% ulna \\
\hline completo & 34,78 & 29,58 & 16,18 & 8,89 \\
proximal & 37,68 & 9,86 & 32,35 & 86,67 \\
distal & 10,14 & 46,48 & 27,94 & 2,22 \\
diáfisis & 17,39 & 14,08 & 23,53 & 2,22 \\
\hline
\end{tabular}

El grado de digestión evaluado sobre dientes, fémures y húmeros se muestra en la Tabla 4. Cabe destacar que no se reconocieron diferencias significativas en las evidencias de digestión que se puedan vincular con cada uno de los taxones presentes en la asociación (Figura 4).

Tabla 4. Grados de digestión en elementos postcraneanos y dientes de la asociación del Quequén Salado.

Table 4. Degree of digestion of postcranial elements and teeth present in Quequén Salado assemblage.

\begin{tabular}{ccccc}
\hline & $\%$ nula o leve & $\%$ moderada & $\%$ fuerte & $\%$ extrema \\
\hline fémur & 64 & 22 & 12 & 2 \\
húmero & 79,63 & 12,96 & 7,41 & 0 \\
incisivo aislado & 80,36 & 12,50 & 3,57 & 3,57 \\
incisivo in situ & 85,96 & 14,04 & 0 & 0 \\
molar aislado & 100 & 0 & 0 & 0 \\
molar in situ & 100 & 0 & 0 & 0 \\
\hline
\end{tabular}

Los cortes delgados realizados sobre una de las acumulaciones mostraron una densidad ósea muy alta. Son claramente observables metapodios enteros y rotos, en los que se pudieron identificar los discos epifisarios, las epífisis y la cavidad medular. Algunos metapodios estaban alineados entre sí, manteniendo su posición anatómica original. También se observaron diáfisis de huesos largos, un incisivo aislado de roedor y una mandíbula de Ctenomys sp. con el incisivo y todos los molares, pero sin la región articular. Además, se registraron varios restos esqueléticos indeterminables. En los dientes incluidos en esta acumulación no se observaron evidencias marcadas de corrosión por ácidos digestivos. 

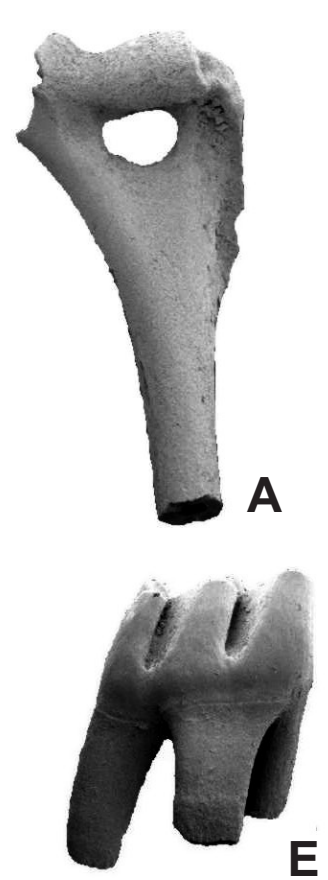
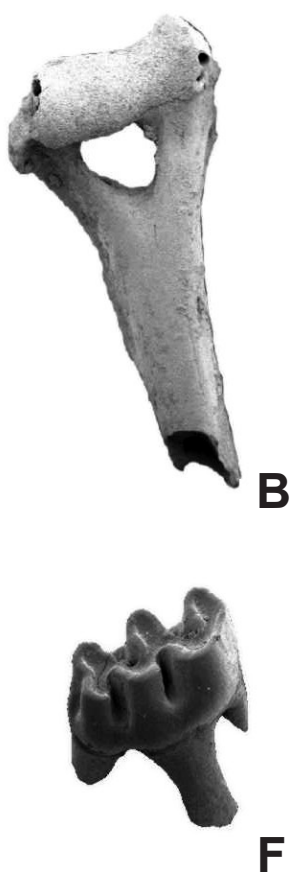

$\mathbf{F}$
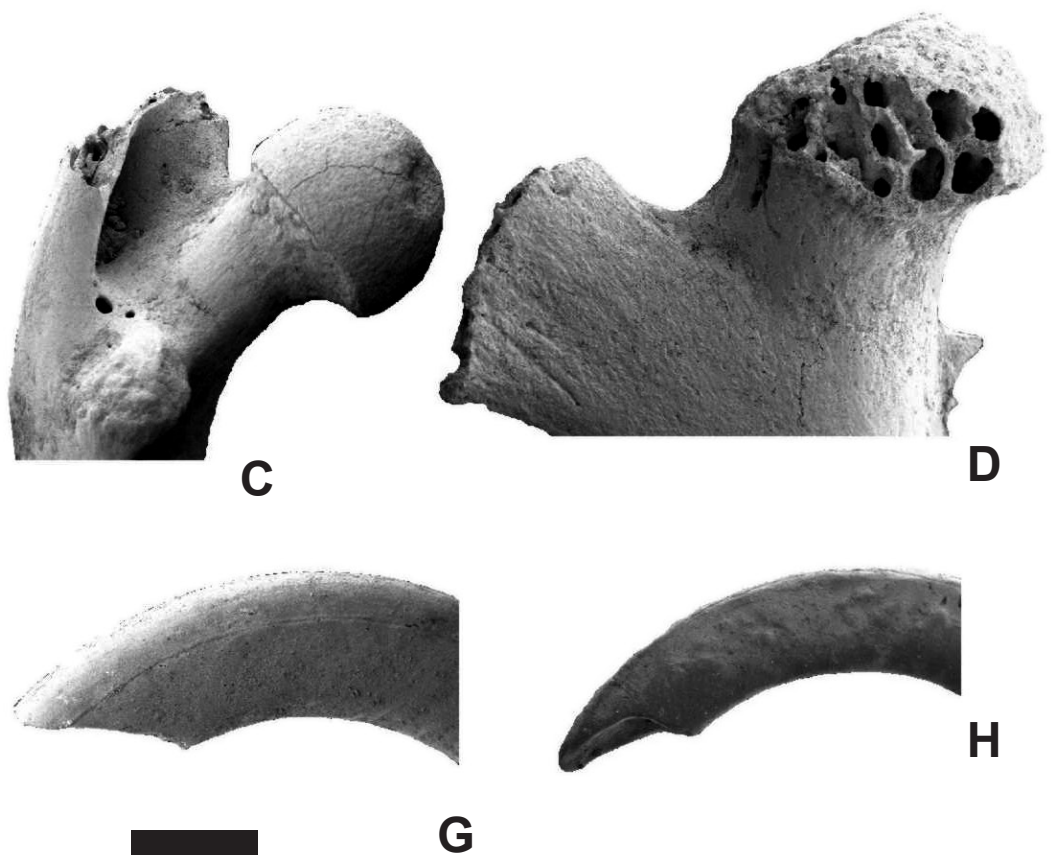

Figura 4. A, porción distal de húmero con grado de digestión leve; $\mathbf{B}$, porción distal de húmero con grado de digestión moderada; C, porción proximal de fémur sin evidencias de digestión; $\mathbf{D}$, porción proximal de fémur con grado de digestión extrema; $\mathbf{E}-\mathbf{F}$, molares aislados sin evidencias de digestión; G, incisivo con grado de digestión leve; $\mathbf{H}$, incisivo con grado de digestión moderada. Escala = 1 mm.

Figure 4. A, distal humerus with light degree of digestion; B, distal humerus with moderate degree of digestion; C, proximal femur without evidence of digestion; D, proximal femur with extreme degree of digestion; E-F, isolated molars without evidence of digestion; G, incisor with light degree of digestion; $\mathbf{H}$, incisor with moderate degree of digestion. Scale bar $=1 \mathrm{~mm}$.

En cuanto a las características microestructurales (Figuras $5 \mathrm{~A}, \mathrm{C})$ no se observaron estructuras asignables a osteonas organizadas. Sin embargo, se verificó la presencia de los espacios donde se alojaban los osteocitos (osteoplastos). Estas lagunas son fusiformes, están distribuidas de manera uniforme, y entre ellas se observan finos canalículos, correspondientes a los canales calcóforos, que constituyen las zonas donde se ubicaban las prolongaciones citoplasmáticas de los osteocitos. También se determinaron conductos vasculares. En las dos secciones delgadas analizadas se reconoció el desarrollo de microfisuras con direcciones variables, que atravesaban la microestructura ósea.

Tanto las cavidades, originadas por la pérdida de la materia orgánica, como las microfisuras están rellenas principalmente por calcita esparítica, en algunos casos en forma de drusas, $\mathrm{y}$, en menor medida, por óxidos de hierro y manganeso (Figuras 5A,B). En ninguno de los cortes se observaron evidencias de bioerosión. La matriz que contiene los fósiles es arcillosa e incluye diferentes minerales, como cuarzo, calcita, plagioclasas, trizas de vidrio volcánico, óxidos de hierro y óxidos de manganeso y fragmentos líticos volcánicos (Figura 5D), en concordancia con la composición volcaniclástica de los depósitos que constituyen la roca hospedante de dichos restos.

Los diagramas de composición química elemental (EDX) evidenciaron la presencia de $\mathrm{P}$ y $\mathrm{Ca}$ en todos los elementos esqueléticos fósiles analizados. Además, en ellos también se registraron $\mathrm{Na}, \mathrm{Mg}, \mathrm{Al}$, Si y Fe (Figura 6A). A excepción del $\mathrm{P}$, el resto de los elementos químicos fueron identificados tanto en la matriz que contenía los restos (Figura 6B) como en la roca hospedante (Figura 6C).

\section{DISCUSIÓN}

Sobre la base de su forma, tamaño, densidad ósea y caracteres generales de los elementos esqueléticos conservados, las acumulaciones analizadas se interpretaron como egagrópilas fósiles. En cuanto a los restos que se encontraban aislados pero asociados a estas acumulaciones, su proximidad espacial en el afloramiento y sus características intrínsecas (atributos tafonómicos y taxonómicos) (Pardiñas, 1999), permitieron considerarlos como procedentes de egagrópilas. Probablemente la desagregación se habría producido en etapas cercanas al enterramiento o durante el mismo, ya que ningún resto muestra evidencias de meteorización por exposición subaérea.

Trejo \& Ojeda (2002) registraron valores promedio en las egagrópilas de Tyto alba (Scopoli, 1769) (Strigiformes) de 4,19 $\mathrm{cm}$ de largo máximo (rango de 2,60 y 7,30 cm) y 2,70 cm de ancho máximo (rango de 1,85 y 4,14 cm), mientras que para las de Bubo virginianus (Gmelin, 1788) (Strigiformes) los valores fueron de $4,46 \mathrm{~cm}$ (rango de 3,06 y $6,40 \mathrm{~cm}$ ) y $2,83 \mathrm{~cm}$ (rango de 2,46 y $2,93 \mathrm{~cm}$ ) respectivamente. Estos autores observaron una superposición de tamaños en las egagrópilas de varias de las especies de rapaces estudiadas e indicaron que T. alba y $B$. virginianus formarían parte del grupo que producen egagrópilas más grandes. Para el caso de Athene cunicularia (Molina, 1782) (Strigiformes), Montalvo \& Tejerina (2009) indicaron valores 

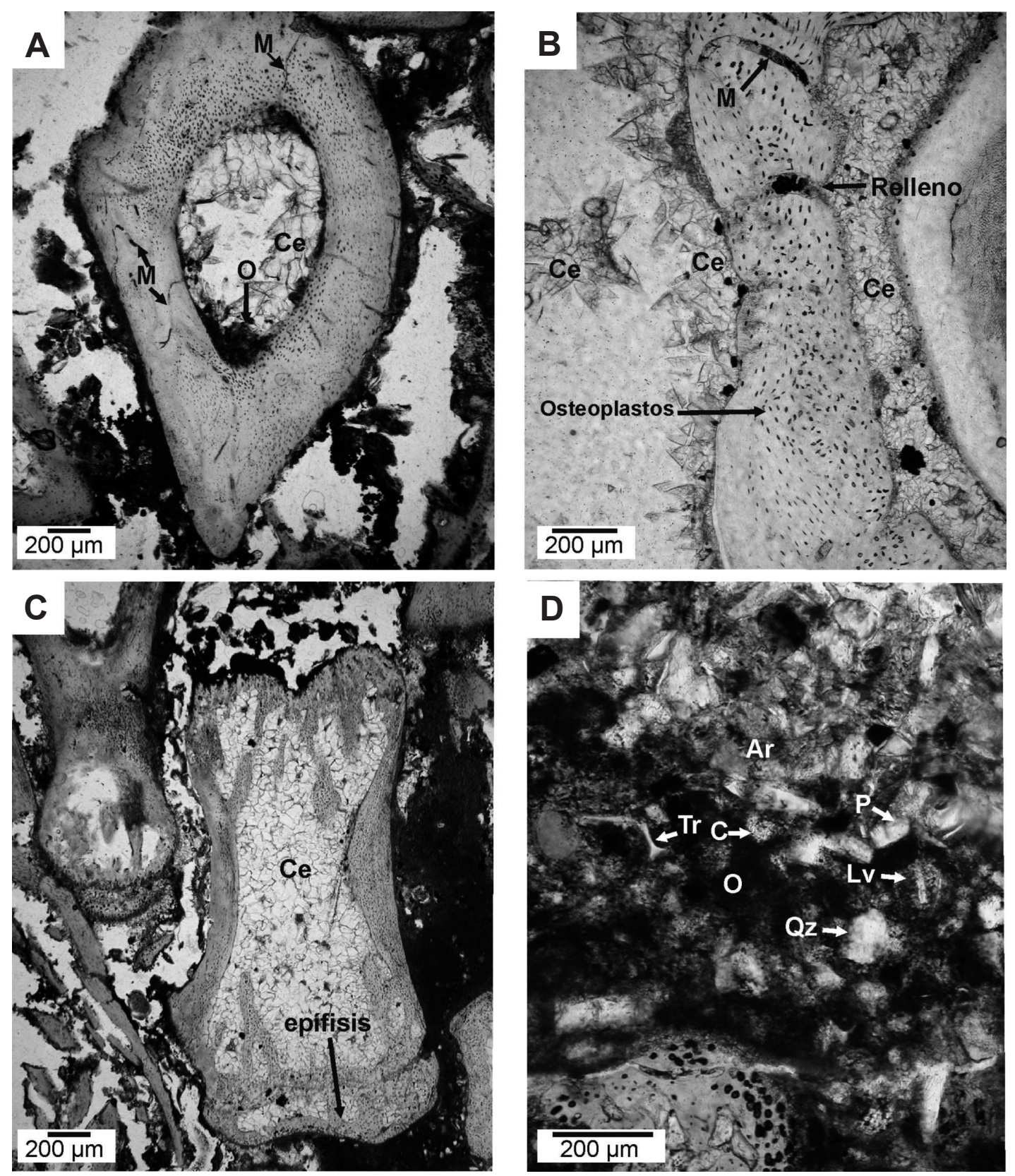

Figura 5. A, corte de diáfisis con microfisuras (M) rellena con calcita esparítica (Ce) y óxidos de Fe/Mn (O); B, hueso indet. con microfisuras (M) rellenas por óxidos y cristales de calcita esparítica $(\mathbf{C e})$; C, metapodio relleno por calcita esparítica $(\mathbf{C e})$; $\mathbf{D}$, detalle del sedimento compuesto por arcilla $(\mathbf{A r})$, cuarzo $(\mathbf{Q z})$, plagioclasas $(\mathbf{P})$, trizas vítreas $(\mathbf{T r})$, fragmentos líticos volcánicos $(\mathbf{L v})$, calcita $(\mathbf{C})$ y óxidos de Fe/Mn $(\mathbf{O})$.

Figure 5. A, shaft indet. with microcracks (M) filled with sparry calcite $(\mathbf{C e})$ and Fe/Mn oxides $(\mathbf{O})$; $\mathbf{B}$, bone indet. with microcracks (M) filled with sparry calcite crystals and oxides (Ce); C, metapodial filled by sparry calcite (Ce); D, detail of sediment composed of clay (Ar), quartz (Qz), plagioclase (P), glass shards (Tr), volcanic lithic fragments (Lv), calcite (C) and oxides of Fe/Mn (O).

promedio de $3,58 \mathrm{~cm}$ de largo máximo y $1,51 \mathrm{~cm}$ de ancho máximo. Las medidas de las egagrópilas analizadas en este trabajo son coherentes con las de las egagrópilas correspondientes a las rapaces nocturnas mencionadas.

La Figura 7 muestra los porcentajes obtenidos para los diferentes elementos esqueléticos identificados. Resultó alta la frecuencia de vértebras y metapodios, elementos que son abundantes en los esqueletos. En cuanto a los demás, sólo fue un poco más alta la frecuencia de fragmentos craneanos, lo que indicaría un alto grado de rotura de los cráneos. Considerando toda la asociación, el 21\% de los elementos evaluados correspondía a porciones craneanas (paladares, mandíbulas, fragmentos craneanos y dientes aislados). Al evaluar los restos contenidos en egagrópilas de Athene cunicularia recuperadas cerca de la boca de una cueva, Montalvo et al. (2012b) encontraron que el $25 \%$ de los identificados correspondía a elementos craneanos. Por otro lado, al considerar todos los restos aislados hallados alrededor de la misma cueva, los elementos craneanos representaban el 30\% del total. El valor obtenido en la muestra del Quequén Salado es apenas más bajo que el evaluado para egagrópilas actuales de $A$. cunicularia y mucho más bajo que el de los restos dispersos, lo que podría 

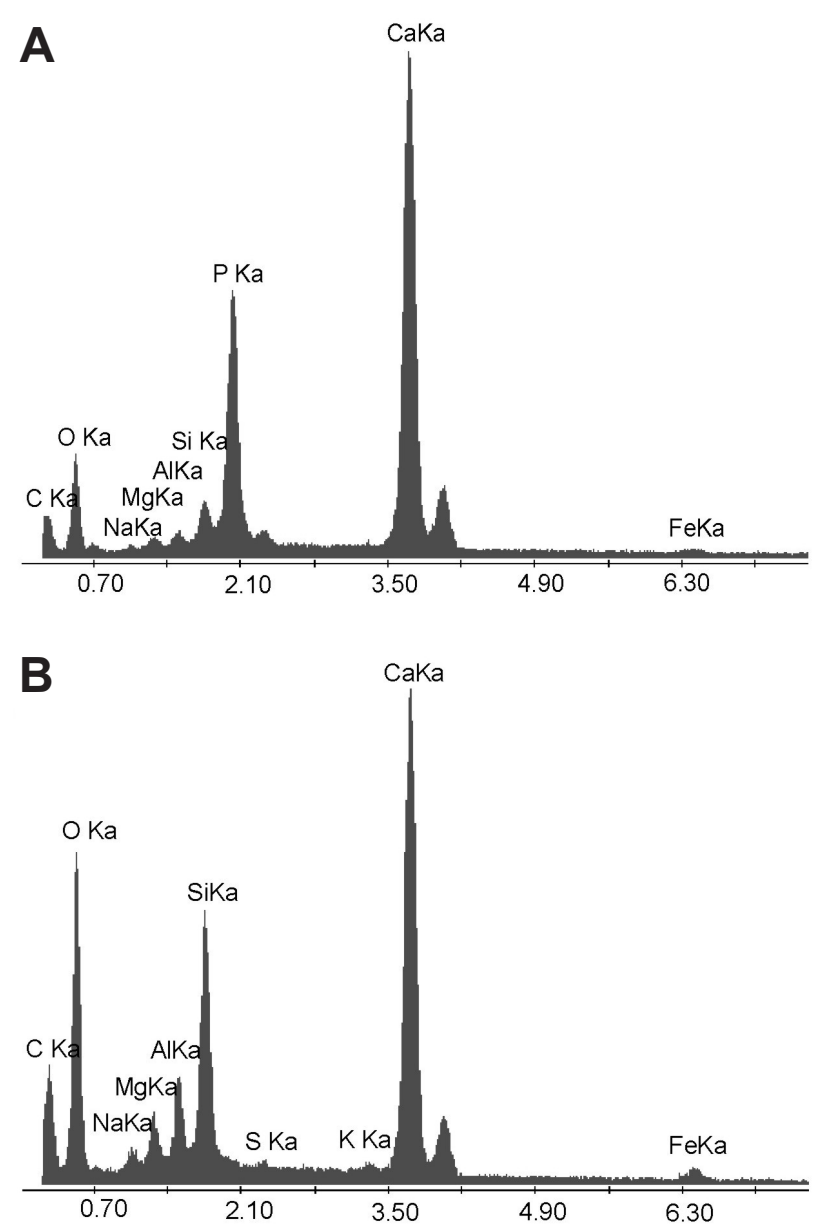

C

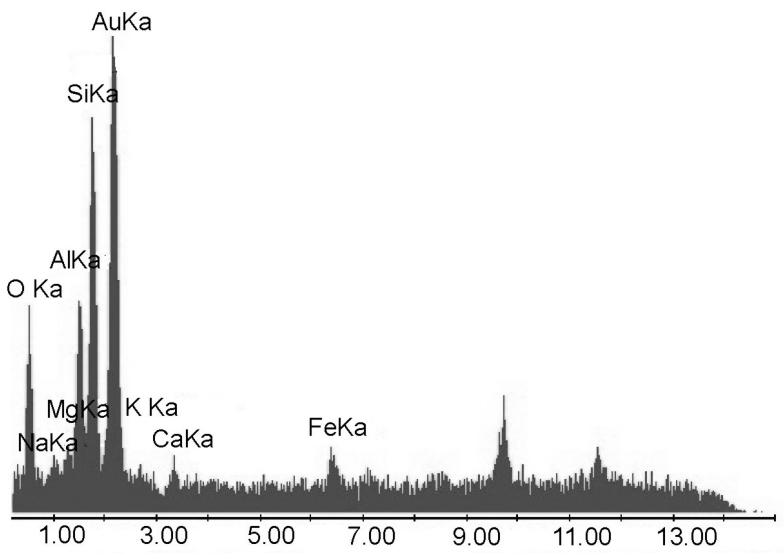

Figura 6. Análisis de EDX. A, resto contenido en la egagrópila; B, matriz de la egagrópila; $\mathbf{C}$, roca hospedante.

Figure 6. EDX analysis. A, pellet's remains; B, pellet's matrix; C, hosted rock.

indicar que la acumulación original se mantuvo sin mucha pérdida o transporte de elementos esqueléticos. La curva de frecuencia de elementos obtenida resultó semejante a la propuesta por Andrews (1990) para rapaces nocturnas. A su vez, este autor también destacó que para Tyto alba la distribución de elementos esqueléticos es prácticamente similar a la ocurrencia natural de huesos en los esqueletos de mamíferos.

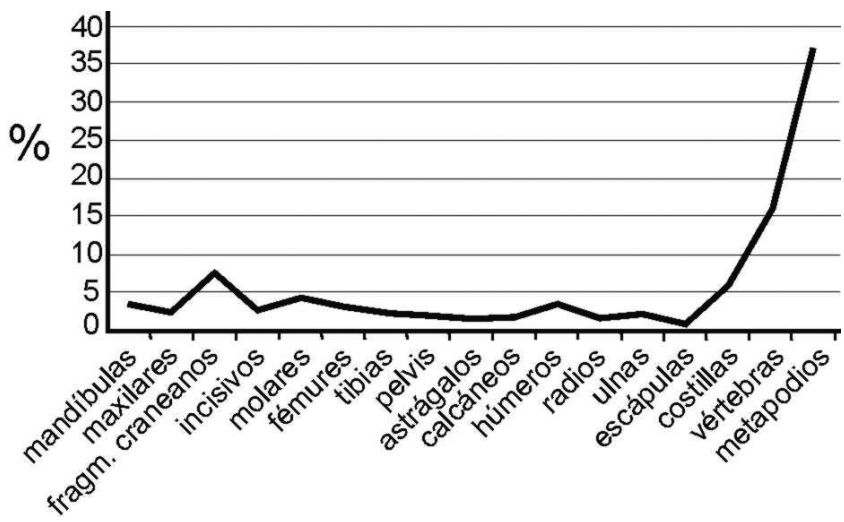

Figura 7. Proporción de elementos esqueléticos expresada en porcentaje en la asociación del Quequén Salado.

Figure 7. Skeletal elements proportion expressed as percentage in the Quequén Salado assemblage.

El promedio de la abundancia relativa de los diferentes elementos esqueléticos preservados fue alto $(54,81 \%)$. En ese sentido, para egagrópilas de Tyto alba, Andrews (1990) obtuvo promedios que fluctúan entre $27,20 \%$ y $63,20 \%$ (promedio $=45,23 \%$ ). Por su parte, Gómez (2007) indicó un valor de $59,87 \%$ para una muestra de esta misma especie proveniente de la Región Pampeana. Para Athene cunicularia, Gómez (2007) obtuvo un promedio de 35,45\% y Montalvo \& Tejerina (2009) de 55,62\%, en ambos casos a partir de muestras procedentes de la Región Pampeana. Sobre muestras de Bubo virginianus, recolectadas en localidades de las provincias de Catamarca y Buenos Aires, Gómez (2005) obtuvo un promedio de $58,30 \%$. Se destaca que, tanto para muestras producidas por aves diurnas como por mamíferos carnívoros, los promedios de abundancia relativa son siempre más bajos que el obtenido en la muestra del Quequén Salado (e.g. Andrews, 1990; Montalvo et al., 2007, 2008, 2012a; Montalvo \& Tallade, 2009, 2010). Si bien Andrews (1990) mencionó que la frecuencia relativa no es suficiente atributo para diferenciar depredadores, es evidente que los resultados obtenidos en ese sentido en la muestra del Quequén Salado son similares a los mencionados para rapaces nocturnas.

Cuando se compara la curva de representación anatómica de la asociación recuperada del Quequén Salado con curvas de muestras actuales producidas por Tyto alba (Andrews, 1990; Gómez, 2007), se observa que en la primera la frecuencia de escápulas, radios y vértebras es menor (Figura 8). Al respecto, escápulas y radios son elementos escasos en otras muestras fósiles del Pleistoceno de Buenos Aires y Chubut y en actuales de T. alba estudiadas por Pardiñas (2004). Gómez (2005) también indicó escasez de escápulas en una muestra de Bubo virginianus. Estos resultados podrían estar vinculados con la fragilidad de dichos elementos, lo que favorecería su rotura. Los elementos mejor representados en la asociación del Quequén Salado fueron las mandíbulas, húmeros y fémures. Los dos primeros incluso están mejor representados que en las muestras mencionadas de T. alba. Por otro lado, la muestra del Quequén Salado difiere de la B. virginianus en los valores obtenidos para pelvis y tibias. Cuando se la compara con la de restos recuperados de egagrópilas de Athene cunicularia se 
observa que para esta especie hay una mejor representación de escápulas, radios y pelvis, en tanto que dientes y metapodios presentan una menor frecuencia (Montalvo \& Tejerina, 2009). De todas maneras, más allá de las diferencias mencionadas, se puede considerar que la curva de representación anatómica de la asociación del Quequén Salado muestra un perfil semejante al descripto por Andrews (1990) como "modelo de lechuzas", que corresponde a aquel obtenido sobre la base de las muestras producidas por rapaces nocturnas.

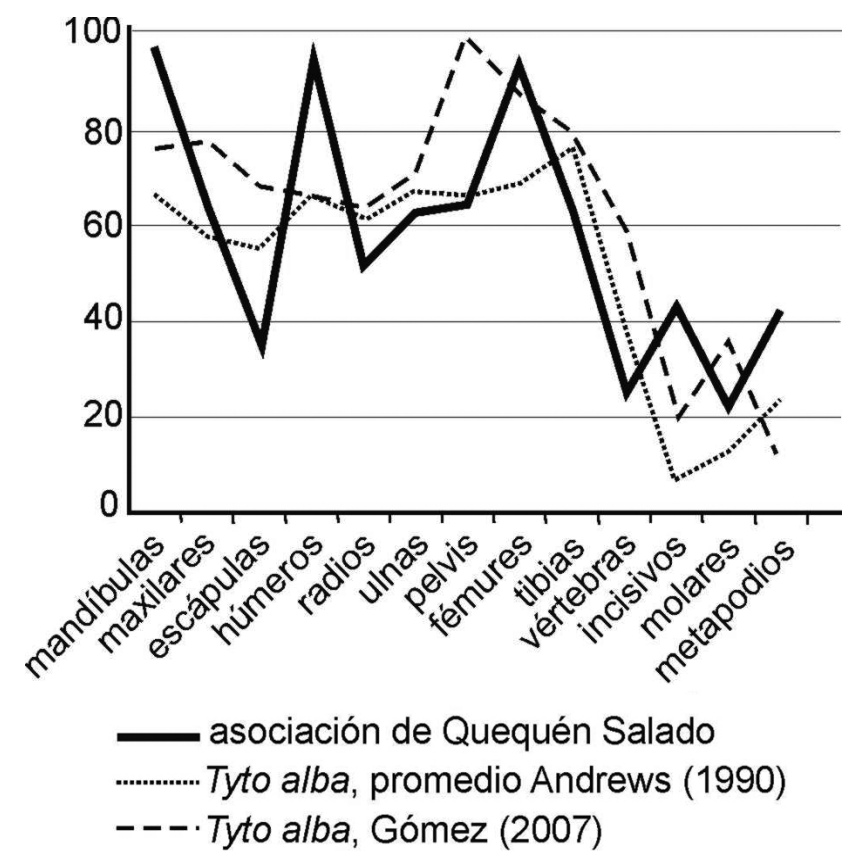

Figura 8. Promedio de abundancia relativa de los elementos esqueléticos de la asociación del Quequén Salado comparados con los datos promedio de asociaciones actuales de Tyto alba tomados de Andrews (1990) y Gómez (2005).

Figure 8. Average relative abundance of skeletal elements from Quequén Salado assemblage compared with mean data for Tyto alba from Andrews (1990) and Gómez (2005).

Los índices que evalúan proporciones de elementos craneanos y postcraneanos indican una leve deficiencia de los primeros. En relación a ello, Andrews (1990) indicó que para rapaces nocturnas el número de elementos craneanos y postcraneanos es similar. Si bien la leve deficiencia de restos craneanos en la asociación del Quequén Salado se podría vincular con las roturas de esos elementos, los valores obtenidos son semejantes a los propuestos por Gómez (2007) para una muestra actual de Tyto alba.

El índice que evalúa la proporción de elementos proximales y distales de los miembros muestra pérdida de estos últimos, mayor aún que la registrada por Gómez (2005, 2007) en muestras de Bubo virginianus y Tyto alba. Por su parte, Andrews (1990) señaló una leve pérdida de elementos distales en muestras de lechuzas. Las diferencias reconocidas en la muestra del Quequén Salado podrían también estar vinculadas a la rotura posterior al enterramiento de los elementos y la consecuente pérdida de la información.

Los valores obtenidos para los índices de molares e incisivos aislados, superiores a $100 \%$, indican pérdida de mandíbulas y maxilares. Estos valores son muy superiores a los obtenidos por Gómez $(2005,2007)$ para muestras de Bubo virginianus y Tyto alba, y pueden estar vinculados con roturas de los elementos craneanos ocurridas en etapas posteriores al enterramiento y la consecuente preservación de los dientes aislados.

Entre los elementos craneanos, la evaluación de rotura mostró en primer lugar una clara diferencia de preservación vinculada con el taxón y, por lo tanto, con el tamaño de los restos. Los cráneos de los roedores de mayor tamaño, Ctenomys sp., se encontraron menos afectados y varios de ellos conservaban parte del paladar y rostro. En el caso de los sigmodontinos, la rotura fue mayor y resultó muy frecuente el hallazgo de paladares con pérdida de molares. En todos los taxones se identificaron fracturas de borde liso, las cuales se pueden haber producido en cualquier momento posterior a la depredación y, por lo tanto, no brindan información acerca de este proceso. Si bien la fragilidad propia de los elementos craneanos probablemente contribuyó a aumentar la frecuencia de roturas diagenéticas, ciertos caracteres de los mismos pueden estar directamente vinculados con la actividad depredadora. Entre ellos la pérdida de la base craneana, la región occipital y las arcadas cigomáticas. Por otro lado, hubo una alta proporción de mandíbulas con la porción articular rota. En general éstas conservaban todos los molariformes, aunque fue frecuente la pérdida de los incisivos. Gómez (2007) registró una mejor conservación de los cráneos y mandíbulas en muestras de Tyto alba, indicando, por ejemplo, que los cráneos preservaban las arcadas cigomáticas.

La evaluación de los grados de rotura en los elementos postcraneanos (fémures, húmeros, tibias y ulnas) mostró un porcentaje bajo de huesos enteros $(23,72 \%)$. Esta proporción se incrementa cuando se consideran otros elementos como los metapodios, la mayoría de los cuales se preservaron sin roturas. Elementos de mayor fragilidad, como escápulas y pelvis se encontraron siempre rotas; sin embargo, es difícil evaluar si esta rotura está vinculada con la actividad depredadora o fue posterior. Smoke \& Stahl (2004) mencionaron a las escápulas como uno de los elementos que rápidamente se rompen por compactación de los niveles portadores. La relación de huesos largos enteros mencionada es mucho menor a las indicadas por Andrews (1990) para las rapaces nocturnas, quien señaló una proporción alta de restos completos. Con respecto a muestras de Bubo virginianus, Gómez (2005) indicó un porcentaje variable (56\% a 70\%) de elementos enteros. Gómez (2007) encontró que el $96,6 \%$ de los huesos largos evaluados en muestras producidas por Tyto alba estaban enteros, en tanto para muestras de Athene cunicularia el porcentaje fue de 71,4\%. Para esta última especie, Montalvo \& Tejerina (2009) indicaron que el $73,77 \%$ de los huesos largos estaban enteros. Las diferencias encontradas en este análisis muestran una mayor modificación por rotura en la muestra del Quequén Salado respecto de las producidas por rapaces nocturnas. Sin embargo, este atributo es tomado con precaución, ya que entre los restos rotos hubo un porcentaje importante de roturas transversales de borde liso (Marshall, 1989), las que se interpretaron como diagenéticas y que podrían haber afectado, en etapas posteriores a la acumulación, a restos que estaban enteros. 
De acuerdo a los porcentajes obtenidos al evaluar la digestión, esta asociación puede atribuirse a una rapaz nocturna (Andrews, 1990), ya que el 92\% de los elementos esqueléticos evaluados no presentaron evidencias o éstas sólo fueron leves. Se registraron también algunos elementos con digestión moderada (5\%) y el resto con digestión fuerte y extrema. Las evidencias leves, caracterizadas por un ligero punteado, afectaron del mismo modo a los molares e incisivos de los distintos taxones de roedores evaluados. Demirel et al. (2011), al analizar una asociación pleistocénica de Turquía, reconocieron diferencias entre los taxones presentes respecto a las modificaciones producidas por corrosión digestiva. En la asociación del Quequén Salado, si bien los representantes de los taxones registrados (Ctenomys sp. y sigmodontinos) difieren en el tamaño de sus elementos esqueléticos, en función de sus masas corporales estimadas y en la morfología de sus molariformes, no se reconoció ninguna relación directa entre los grados de digestión y el taxón afectado. Tampoco hubo diferencias en los resultados obtenidos para dientes aislados o dientes in situ.

Los incisivos y los extremos proximales de fémures fueron los elementos esqueléticos que tuvieron las modificaciones más importantes (fuertes y extremas); sin embargo, los porcentajes de restos con estas modificaciones fueron muy bajos. Matthews et al. (2006) sugirieron, en base a proporciones similares de incisivos con grados de digestión leve, moderada y fuerte, que la asociación del Pleistoceno de Sudáfrica que estudiaron se podía asignar a Tyto alba. Gómez $(2005,2007)$ obtuvo valores altos de elementos con evidencias de digestión leve en muestras de Tyto alba. Para Bubo virginianus y Athene cunicularia los restos presentaban modificaciones correspondientes a todas las categorías, si bien las leves y moderadas fueron las de mayor frecuencia (Gómez, 2007; Montalvo \& Tejerina, 2009). Por su parte, Montalvo \& Tejerina (2009) señalaron para una asociación producida por A. cunicularia, una alta proporción de restos con evidencias de digestión leves (60\%) y moderadas (30\%), pero con un mayor porcentaje de elementos con evidencias fuertes y extremas que las obtenidas por Gómez (2007).

La evaluación de todos los atributos tafonómicos considerados indica que la asociación del Quequén Salado corresponde a una acumulación producida por una rapaz nocturna (Tabla 5). Si bien el grado de rotura de cráneos y mandíbulas corresponde a categorías de mayor modificación que aquellas que normalmente producen las rapaces nocturnas, en este caso se consideró que ese resultado puede deberse a procesos ocurridos en etapas posteriores a la acumulación.

En relación al registro fósil en la provincia de Buenos Aires de los distintos taxones de rapaces nocturnas consideradas en este trabajo, Tyto alba está presente desde el Pleistoceno temprano (Cenizo \& de los Reyes, 2008), mientras que Athene cunicularia se reconoce desde el Pleistoceno tardío (Tonni, 1983). Por otra parte, no se conocen restos fósiles de Bubo virginianus.

El grado de preservación de los distintos elementos microestructurales reconocidos a partir de los cortes fue muy bueno, lo que permitiría incluir a los ejemplares analizados en las categorías 4 y 5 del índice histológico propuesto por Hedges et al. (1995), es decir con un porcentaje de integridad variable entre $80 \%$ y $95 \%$. La dificultad para identificar osteonas bien desarrolladas estaría vinculada a la región del elemento esquelético observado en cada corte, la dirección del mismo y con la posibilidad de que estos elementos correspondieran a individuos juveniles. La microestructura ósea sólo resultó afectada por el desarrollo de microfisuras y el proceso de permineralización (sensu Fernández-López, 2000).

La calcita esparítica que rellena las microfisuras y los espacios vacíos originados por la pérdida de la materia orgánica en los canales vasculares, lagunas y canalículos sería una evidencia de la precipitación de los carbonatos disueltos durante las inundaciones, reprecipitados en los períodos de formación de los suelos en la llanura de inundación (Jaillard \& Callot, 1987), corroborado por la presencia de rizoconcreciones en el nivel portador. La presencia de óxidos de hierro y manganeso en microfisuras y cavidades se puede vincular con la redistribución de estas sustancias durante la formación del suelo, en respuesta a las fluctuaciones del potencial redox asociado con la saturación periódica de agua (Kemp \& Zárate, 2000). Estos óxidos también sugieren variaciones del nivel de la capa freática en suelos bien drenados (Buol et al., 1991).

Tabla 5. Categorización de la asociación del Quequén Salado de acuerdo a las diferentes características propuestas por Andrews (1990).

Table 5. Categorization of Quequén Salado assemblage according to different analyzed variables proposed by Andrews (1990).

\begin{tabular}{|c|c|c|c|c|c|}
\hline & Categoría 1 & Categoría 2 & Categoría 3 & Categoría 4 & Categoría 5 \\
\hline \multicolumn{6}{|l|}{ Abundancia relativa } \\
\hline \multicolumn{6}{|l|}{ Relación cráneo/ postcráneo } \\
\hline \multicolumn{6}{|l|}{ Pérdida de elementos distales } \\
\hline \multicolumn{6}{|l|}{ Rotura de elementos poscraneanos } \\
\hline \multicolumn{6}{|l|}{ Rotura de elementos craneanos } \\
\hline \multicolumn{6}{|l|}{ Ausencia de arco cigomático } \\
\hline \multicolumn{6}{|l|}{ Pérdida de dientes maxilares } \\
\hline \multicolumn{6}{|l|}{ Rotura de mandíbula } \\
\hline \multicolumn{6}{|l|}{ Pérdida de dientes mandibulares } \\
\hline \multicolumn{6}{|l|}{ Proporción de dientes aislados } \\
\hline \multicolumn{6}{|l|}{ Rotura de dientes } \\
\hline \multicolumn{6}{|l|}{ Digestión en molares } \\
\hline \multicolumn{6}{|l|}{ Digestión en incisivos } \\
\hline Digestión en elementos postcraneales & & & & & \\
\hline
\end{tabular}


De acuerdo a los análisis de EDX se infirió una composición fosfato-cálcica de los restos. Teniendo en cuenta las coincidencias registradas entre la composición química de los materiales fósiles, la matriz que los contenía y los sedimentos portadores, se estima que sólo hubo intercambios de elementos químicos con la roca de caja que conformaba el microambiente donde los huesos fueron enterrados y éstos habrían sido movilizados y aportados por el agua subterránea circundante. Esto avalaría la ausencia de transporte de las egagrópilas una vez que fueron enterradas. La ausencia de fósforo en la matriz de las egagrópilas es un carácter que avala la asignación de las acumulaciones a egagrópilas ya que permite diferenciarlas de los coprolitos (Pollard, 1990; Tomassini \& Montalvo, 2010).

La presencia de egagrópilas completas, la concentración de elementos esqueléticos y las rizoconcreciones descriptas en los niveles portadores avalan la ausencia de reelaboración (sensu Fernández-López, 2000) y homogeneización temporal en la asociación.

Tonni \& Fidalgo (1978) y Alberdi et al. (1989) hicieron referencia a la "Fauna local Quequén Salado - Indio Rico", para agrupar al conjunto de mamíferos fósiles procedentes de las secuencias sedimentarias aflorantes en las cercanías de la confluencia de los ríos Quequén Salado e Indio Rico

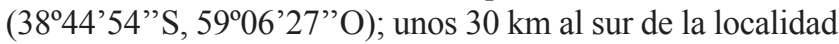
analizada en este trabajo. Los sedimentos portadores fueron correlacionados por estos autores con el Miembro Guerrero de la Formación Lujan (Fidalgo et al., 1973) y asignados a la parte final del Pleistoceno Superior. Por sus características litológicas y estratigráficas, los niveles portadores de la asociación aquí estudiada se pueden correlacionar con aquellos.

De acuerdo al esquema bioestratigráfico propuesto por Cione \& Tonni (2005), para el Cenozoico Superior de la Provincia de Buenos Aires, la especie Macrauchenia patachonica presenta un biocrón que abarca el lapso Bonaerense-Lujanense. Sin embargo, Deschamps (2005) la utilizó para definir, en el sur de la provincia de Buenos Aires, la Biozona de Equus (Amerhippus) neogaeus Macrauchenia patachonica, la cual representaría el Piso/ Edad Lujanense y que, según Cione \& Tonni (2005, p. 195), se "corresponde conceptualmente con la Biozona de Equus (Amerhippus) neogaeus", definida por estos autores como la base bioestratigráfica del Piso/Edad Lujanense.

La asociación faunística aquí estudiada se puede asignar al Pleistoceno Superior y probablemente al Piso/Edad Lujanense (ca. 130-8 ka AP., sensu Cione et al., 2007), principalmente en base a la presencia de Macrauchenia patachonica. El resto de los taxones de microvertebrados reconocidos no contradice esta asignación y, por el contrario, varios de ellos aparecen con frecuencia asociados en depósitos lujanenses de la Provincia de Buenos Aires (Tonni et al., 1985; Bargo et al., 1986; Prado et al., 1987).

Diversos autores (e.g. Tonni et al., 1999, 2003; Quattrocchio et al., 2008; Prado \& Alberdi, 2010) propusieron que, durante el Pleistoceno Superior, en la Región Pampeana habrían imperado condiciones climáticas más áridas y probablemente más frías que las actuales, por un descenso en las temperaturas medias. Los micromamíferos fósiles registrados en esta oportunidad, incluyendo a Ctenomys sp., Eligmodontia sp., Reithrodon auritus y Lestodelphys halli, actualmente constituyen una asociación común en los dominios Patagónico y Central (Ringuelet, 1961). La presencia de dichos taxones, permitiría inferir para esta localidad, asignada tentativamente al Piso/Edad Lujanense, el desarrollo de ambientes abiertos de estepas y pastizales, dominadas por vegetación xerófila, con condiciones áridas o semiáridas y probablemente algo más frías que las actuales (Tonni et al., 1988; Pardiñas, 1995; Vucetich \& Verzi, 1995; Goin, 1995, 2001; Prado et al., 1985).

Teniendo en cuenta que Lestodelphys halli es considerada una especie climático-sensitiva (Prado et al., 1985), Prado \& Alberdi (1999) destacaron que constituye uno de los mejores indicadores paleoambientales para la Región Pampeana. La baja riqueza específica de sigmodontinos, registrada en otra localidad del Pleistoceno de la Provincia de Buenos Aires, fue vinculada por Pardiñas (2001) con condiciones de aridez regional. Según Prado et al. (1987), las mayores frecuencias de Reithrodon auritus en egagrópilas de aves rapaces nocturnas ocurren en este tipo de ambiente, donde es fácilmente depredado. Politis \& Tonni (1982) destacaron la frecuencia relativamente alta de $R$. auritus en un sitio arqueológico de la Región Pampeana e indicaron que esto podría atribuirse a condiciones locales más áridas que las de las áreas circundantes.

\section{CONCLUSIONES}

Por sus características, la asociación de microvertebrados estudiada se atribuyó a una acumulación correspondiente a egagrópilas tanto enteras como desagregadas. Si bien a partir de la información disponible resulta difícil ajustar a nivel taxonómico a su productor, el análisis de la representación anatómica y de los grados de digestión muestra claras afinidades con los resultados obtenidos sobre acumulaciones actuales producidas por rapaces nocturnas. Sin embargo, el grado de rotura es el atributo que más sesga esta asociación, ya que mostró valores mucho más altos que aquellos obtenidos en evaluaciones de rapaces nocturnas actuales. En este sentido, es probable que las fracturas de borde liso, comunes entre los elementos esqueléticos recuperados, sean el resultado de roturas producidas en algún momento posterior al enterramiento o directamente durante la extracción de la muestra. De este modo, esas fracturas enmascararían roturas previas producidas al momento de la depredación.

La buena preservación de las acumulaciones y de las características microestructurales de los restos que en ellas se conservaron son indicadoras de un enterramiento rápido. Los restos aislados pero asociados a esas acumulaciones, interpretados como desprendidos de egagrópilas desagregadas, también habrían sido rápidamente enterrados ya que no presentan evidencias de meteorización.

Desde el punto de vista bioestratigráfico, sobre la base de la composición taxonómica registrada y la fauna de megamamíferos asociada, la asociación fósil estudiada, procedente de niveles de llanura de inundación en las barrancas del río Quequén Salado, se asigna al Piso/Edad Lujanense, correspondiente al Pleistoceno Superior. 


\section{AGRADECIMIENTOS}

Este trabajo fue financiado mediante un proyecto desarrollado en la Facultad de Ciencias Exactas y Naturales, Universidad Nacional de La Pampa, La Pampa, Argentina. Agradecemos al Museo Municipal de Ciencias Naturales "Vicente Di Martino" por el acceso a los materiales estudiados. A V. Sorrivas por su ayuda con las fotografías de microscopio electrónico y análisis de EDX y a H. Ortiz por su colaboración con los cortes delgados. Finalmente, agradecemos a los dos revisores y a la editora A.M.Ribeiro, quienes con sus sugerencias ayudaron a mejorar este trabajo.

\section{REFERENCIAS}

Alberdi, M.T.; Menegaz, A.N.; Prado, J.L. \& Tonni, E.P. 1989. La Fauna local Quequén Salado-Indio Rico (Pleistoceno tardío) de la provincia de Buenos Aires, Argentina. Aspectos paleoambientales y bioestratigráficos. Ameghiniana, 25:225-236.

Andrews, P. 1990. Owls, caves and fossils. Predation, preservation, and accumulation of small mammal bones in caves, with the analysis of the Pleistocene cave faunas from Westbury-subMendip, Somerset, UK. Chicago, University Press, 231 p.

Bargo, M.S.; Menegaz, A.N.; Prado, J.L.; Salemme, M.; Tambussi, C. \& Tonni, E.P. 1986. Una nueva fauna local de la Unidad Mamífero Lujanense (Pleistoceno tardío) de la provincia de Buenos Aires. Ameghiniana, 23:229-232.

Behrensmeyer, A.K. \& Hook, R.W. 1992. Paleoenvironmental contexts and taphonomic modes. In: Behrensmeyer, A.K.; Damuth, J.D.; DiMichele, W.A.; Potts, R.; Sues, H. \& Wings, S.L. (eds.) Terrestrial ecosystems through time: evolutionary paleoecology of terrestrial plants and animals. Chicago, University Press, p. 15-136.

Bennàsar Serra, M.L. 2010. Tafonomía de micromamíferos del Pleistoceno Inferior de la Sierra de Atapuerca (Burgos): la Sima del Elefante y la Gran Dolina. Universitat Rovira i Virgili, Tesis Doctoral, $646 \mathrm{p}$.

Buol, S.W.; Hole, F.D. \& Mac Cracken, R.J., 1991. Génesis y clasificación de suelos. México, Editorial Trillas, 417 p.

Cenizo, M.M. \& de los Reyes, L.M. 2008. Primeros registros de Tyto alba (Scopoli, 1769) (Strigiformes, Aves) en el Pleistoceno Medio-Tardío de la provincia de Buenos Aires (Argentina) y sus implicancias tafonómicas. Revista del Museo Argentino de Ciencias Naturales, 10:199-209.

Cione, A.L. \& Tonni, E.P. 2005. Bioestratigrafía basada en mamíferos del Cenozoico superior de la provincia de Buenos Aires, Argentina. In: R.E. de Barrio; R.O. Etcheverry; M.F. Caballé \& E. Llambías, (eds.) Geología y recursos minerales de la provincia de Buenos Aires, p.183-200.

Cione, A.L.; Tonni, E.P.; Bargo, S.; Bond, M.; Candela, A.M.; Carlini, A.A.; Deschamps, C.M.; Dozo, M.T.; Esteban, G.; Goin, F.J.; Montalvo, C.I.; Nasif, N.; Noriega, J.I.; Ortiz Jaureguizar, E.; Pascual, R.; Prado, J.L.; Reguero, M.A.; Scillato-Yané, G.J.; Soibelzon, L.; Verzi, D.H.; Vieytes, C.; Vizcaíno, F. \& Vucetich, M.G. 2007. Mamíferos continentales del Mioceno tardío a la actualidad en la Argentina: cincuenta años de estudios. Ameghiniana, Publicación Especial, 11:257-278.

Demirel, A.; Andrews, P.; Yalçinkaya, I. \& Ersoy, A. 2011. The taphonomy and palaeoenvironmental implications of the small mammals from Karain Cave, Turkey. Journal of Archaeological Science, 38:3048-3059. doi:10.1016/j.jas.2011.07.003
Deschamps, C. 2005. Late Cenozoic mammal bio-chronostratigraphy in southwestern Buenos Aires province, Argentina. Ameghiniana, 42:733-750.

Fernández-Jalvo, Y. \& Andrews, P. 1992. Small mammal taphonomy of Gran Dolina, Atapuerca (Burgos), Spain. Journal of Archaeological Science, 19:407-428. doi:10.1016/03054403(92)90058-B

Fernández-López, S. 2000. Temas de Tafonomía. Departamento de Paleontología. Madrid, Universidad Complutense, 167 p.

Fidalgo, F.; Colado, U. \& De Francesco, R. 1973. Sobre ingresiones marinas cuaternarias en los partidos de Castelli, Chascomús y Magdalena (Provincia de Buenos Aires). In: CONGRESO GEOLÓGICO ARGENTINO, 5, 1975. Actas, Carlos Paz, v. 3, p. 227-240.

Frenguelli, J. 1928. Observaciones geológicas en la región costanera sur de la Provincia de Buenos Aires. Anales de la Facultad de Ciencias Educacionales de Paraná, 2:1-145.

Goddard, E.; Trask, P.; De Ford, R.; Rove, O.; Singewald, J. \& Overbeck, R. 1948. Rock Color Chart. Washington, National Research Council, 8 p.

Goin, F.J. 1995. Los marsupiales. In: M.T. Alberdi; G. Leone \& E.P. Tonni (eds.) Evolución biológica y climática de la Región Pampeana durante los últimos cinco millones de años. Un ensayo de correlación con el Mediterráneo Occidental. Madrid, Museo de Ciencias Naturales de Madrid, Consejo de Investigaciones, p. 165-179 (Monografias 12).

Goin, F.J. 2001. Marsupiales (Didelphidae: Marmosinae y Didelphinae). In: D. Mazzanti \& C. Quintana (eds.) Cueva Tixi: Cazadores y recolectores de las sierras de Tandilia Oriental. l. Geología, Paleontología y Zooarqueología. Mar del Plata, Laboratorio de Arqueología, Universidad Nacional de Mar del Plata, p. 75-114 (Publicación Especial 1).

Gómez, G.N. 2005. Analysis of bone modifications of Bubo virginianus' pellets from Argentina. Journal of Taphonomy, 3:1-16.

Gómez, G. 2007. Predators categorization based on taphonomic analysis of micromammals bones: a comparison to proposed models. In: M.A. Gutierrez; L. Miotti; G. Barrientos; G. Mengoni Goñalons \& M. Salemme (eds.) Taphonomy and Zooarchaeology in Argentina, BAR S1601 (British Archaeological Report), 89-103.

Gómez, G.N. \& Kaufmann, C.A. 2007. Taphonomic analysis of Pseudalopex griseus (Gray, 1837) scat assemblages and their archaeological implications. Journal of Taphonomy, 5:59-70.

Hedges, R.E.M.; Millard, A.R. \& Pike, A.W.G. 1995. Measurements and relationship of diagenetic alteration of bone from three archaeological sites. Journal of Archaeological Science, 22:201209. doi.org/10.1006/jasc.1995.0022

Jaillard, B. \& Callot, G. 1987. Action des racines sur la ségrégation minéralogique des constituants du sol. In: N. Fedoroff; L.M. Bresson \& M.A. Court (eds.) Micromorphologie des sols, Réunion Internationale de Micromorphologie des Sols, p. 371-375.

Kemp, R.A. \& Zárate, M.A. 2000. Pliocene pedosedimentary cycles in the southern Pampas, Argentina. Sedimentology, 47:3-14.

Marshall, L. 1989. Bone modification and "The laws of burial". In: R. Bonnichsen \& M. Sorg (eds.) Bone modification, People of the Americas Publications/University of Main, $535 \mathrm{p}$.

Matthews, T.; Parkington, J.E. \& Denys, C. 2006 The taphonomy of micromammals from the late middle Pleistocene site of Hoedjiespunt 1 (Cape province, south Africa). Journal of Taphonomy, 4:1-16. 
Montalvo, C.I.; Bisceglia, S.; Kin, M. \& Sosa, R.A. 2012a. Taphonomic analysis of rodent bone accumulations produced by Geoffroy's cat (Leopardus geoffroyi, Carnivora, Felidae) in Central Argentina. Journal of Archaeological Science, 39:19331941. doi:10.1016/j.jas.2012.02.024

Montalvo, C.I.; Cheme Arriaga, L.; Tallade, P.O. \& Sosa, R.A. 2012b. Owl pellet dispersal by wind: observations and experimentations. Quaternary International, 278:63-70. doi:10.1016/j.quaint.2012.01.027

Montalvo, C.I.; Pessino, M.E. \& Bagatto, F.C. 2008. Taphonomy of the bones of rodents consumed by Andean hog-nosed skunks (Conepatus chinga, Carnivora, Mephitidae) in central Argentina. Journal of Archaeological Science, 35:1481-1488. doi:10.1016/j. jas.2007.10.011

Montalvo, C.I.; Pessino, M. \& González, V. 2007. Taphonomic analysis of remains of mammals eaten by pumas (Puma concolor Carnivora, Felidae) in central Argentina. Journal of Archaeological Science, 34:2151-2160. doi:10.1016/j. jas.2007.02.012

Montalvo, C.I. \& Tallade, P. 2009. Taphonomy of the accumulations produced by Caracara plancus (Falconidae). Analysis of prey remains and pellets. Journal of Taphonomy, 7:235-248.

Montalvo, C.I. \& Tallade, P.O. 2010. Análisis tafonómico de restos no ingeridos de roedores presa de Caracara plancus (Aves, Falconidae) In: M. De Nigris; P.M. Fernández; M. Giardina; A.F. Gil; M.A. Gutiérrez; A. Izeta; G. Neme \& H.D. Yacobaccio (eds.) Zooarqueología a principios del siglo XXI: aportes teóricos, metodológicos y casos de estudio, Ediciones Libros del Espinillo, p. 419-428.

Montalvo, C. I. \& Tejerina, P. 2009. Análisis tafonómico de los huesos de anfibios y roedores depredados por Athene cunicularia (Strigiformes, Strigidae) en La Pampa, Argentina. In: M. Berón; L. Luna; M. Bonomo, C.I. Montalvo; C. Aranda \& M. Carrera Aizpitarte (eds.) Mamül Mapu: pasado y presente desde la arqueología pampeana, Ediciones Libros del Espinillo, 1:323334.

Ortiz, P.E. \& Pardiñas, U.F.J. 2001. Sigmodontinos (Mammalia: Rodentia) del Pleistoceno Tardío del valle de Tafí (Tucumán, Argentina): taxonomía, tafonomía y reconstrucción paleoambiental. Ameghiniana, 38:3-26.

Pardiñas, U.F.J. 1995. Los roedores cricétidos. In: M.T. Alberdi; G. Leone \& E.P. Tonni (eds.) Evolución biológica y climática de la Región Pampeana durante los últimos cinco millones de años. Un ensayo de correlación con el Mediterráneo Occidental. Madrid, Museo Nacional de Ciencias Naturales de Madrid, p. 229-256 (Monografias 12).

Pardiñas, U.F.J., 1999. Los roedores muroideos del Pleistoceno Tardio-Holoceno en la Región Pampeana (sector este) y Patagonia (República Argentina): aspectos taxonómicos, importancia bioestratigráficas y significación paleoambiental. Facultad de Ciencias Naturales y Museo, Universidad Nacional de La Plata, Tesis Doctoral, 283 p.

Pardiñas, U.F.J. 2001. Condiciones áridas durante el Holoceno Temprano en el sudoeste de la provincia de Buenos Aires (Argentina): vertebrados y tafonomía. Ameghiniana, 38:227-236.

Pardiñas, U.F.J. 2004. Roedores sigmodontinos (Mammalia: Rodentia: Cricetidae) y otros micromamíferos como indicadores de ambientes hacia el Ensenadense cuspidal en el sudeste de la provincia de Buenos Aires (Argentina). Ameghiniana, 41:437-450.

Pardiñas, U.F.J.; Gelfo, J.N.; San Cristóbal, J.; Cione, A.L. \& Tonni, E.P. 1996. Una asociación de organismos marinos y continentales en el Pleistoceno Superior en el sur de la provincia de Buenos Aires, Argentina. In: CONGRESO GEOLÓGICO ARGENTINO, 13/ CONGRESO DE EXPLORACIÓN DE HIDROCARBUROS, 3, 1996. Actas, Buenos Aires, v. 5, p. 95-111.

Politis, G.G. \& Tonni, E.P. 1982. Arqueología de la región pampeana: el sitio 2 de Zanjón Seco (partido de Necochea, provincia de Buenos Aires, Rep. Argentina). Revista de Pré-Historia, Universidad de São Paulo, 3:109-139.

Pollard, J.E. 1990. Evidence for diet. In: D.E.G. Briggs \& P.R. Crowther (eds.) Paleobiology - a synthesis. Blackwell.

Prado, J.L. \& Alberdi, M.T. 1999. The mammalian record and climatic change over the last 13,000 years in the Pampean Region, Argentina. Quaternary International, 57-58:165-174. doi:10.1016/S1040-6182(98)00057-3

Prado, J.L. \& Alberdi, M.T. 2010. Quaternary mammalian faunas of the Pampean Región. Quaternary International, 212:176-186. doi:10.1016/j.quaint.2009.03.010

Prado, J.L.; Goin, F.J. \& Tonni, E.P. 1985. Lestodelphys halli (Mammalia, Didelphidae) in Holocene deposits of southeastern Buenos Aires province, Argentina. Morphological and palaeoenvironmental considerations. Quaternary of South American and Antarctic Peninsula, 3:93-106.

Prado, J.L.; Menegaz, A.; Tonni, E.P. \& Salemme, M., 1987. Los mamíferos de la fauna local Paso Otero (Pleistoceno tardío), provincia de Buenos Aires. Aspectos paleoambientales y bioestratigráficos. Ameghiniana, 24:217-233.

Quattrocchio, M.E.; Borromei, A.M.; Deschamps, C.M.; Grilla, S.C. \& Zavala, C.A. 2008. Landscape evolution and climate changes in the Late Pleistocene-Holocene, southern Pampa (Argentina): evidence from palynology, mammals and sedimentology. Quaternary International, 181:123-138. doi:10.1016/j. quaint.2007.02.018

Ringuelet, R.A. 1961. Rasgos fundamentales de la zoogeografía de la República Argentina. Physis, 22:151-170.

Schillizzi, R.; Gutierrez Tellez, B. \& Aramayo, S. 2006. Reconstrucción paleoambiental del Cuaternario en las barrancas del río Quequén Salado, provincia de Buenos Aires, Argentina. In: CONGRESO ARGENTINO DE CUATERNARIO Y GEOMORFOLOGÍA, 3, 2006. Actas, Córdoba, v. 2, p. 649-658.

Smoke, N.D. \& Stahl, P.W. 2004. Post-burial fragmentation of microvertebrate skeletons. Journal of Archaeological Science, 31:1093-1100. doi:10.1016/j.jas.2004.01.005

Tiranti, S.I. 1992. Barn owl prey in southern La Pampa, Argentina. Journal of Raptor Research, 26:89-92.

Tomassini, R.L. \& Montalvo, C. 2010. Coprolitos en la Formación Monte Hermoso (Mioceno tardío-Plioceno temprano), Farola Monte Hermoso, Buenos Aires, Argentina. Ameghiniana, 47:111-115.

Tonni, E.P. 1983. Aves de un sitio arqueológico del área interserrana de la provincia de Buenos Aires. Ameghiniana, 20:3-10.

Tonni, E.P.; Bargo. M.S. \& Prado, J.L. 1988. Los cambios ambientales en el Pleistoceno tardío y Holoceno del sudeste de la provincia de Buenos Aires a través de una secuencia de mamíferos. Ameghiniana, 25:99-110.

Tonni, E.P.; Cione, A.L. \& Figini, A. 1999. Predominance of arid climates indicated by mammals in the pampas of Argentina during the Late Pleistocene and Holocene. Palaeogeography, Palaeoclimatology, Palaeoecology, 147:257-281. doi:10.1016/ S0031-0182(98)00140-0

Tonni, E.P. \& Fidalgo, F. 1978. Consideraciones sobre los cambios climáticos durante el Pleistoceno tardío-Reciente en la Provincia de Buenos Aires. Aspectos ecológicos y zoogeográficos relacionados. Ameghiniana, 15:235-253. 
Tonni, E.P.; Huarte, R.A.; Carbonari, J.E. \& Figini, A.J. 2003. New radiocarbon chronology for the Guerrero Member of the Luján Formation (Buenos Aires, Argentina): palaeoclimatic significance. Quaternary International, 109-110:45-48. doi:10.1016/S1040-6182(02)00201-X

Tonni, E.P.; Prado, J.L.; Menegaz, A.N. \& Salemme, M.C. 1985. La Unidad Mamífero (Fauna) Lujanense. Proyección de la Estratigrafía mamaliana al Cuaternario de la Región Pampeana. Ameghiniana, 22:255-261.

Trejo, A. \& Ojeda, V. 2002. Identificación de egagrópilas de aves rapaces en ambientes boscosos y ecotonales del noroeste de la Patagonia argentina. Ornitología Neotropical, 13:313-317.
Verzi, D.H.; Montalvo, C.I. \& Deschamps, C.M. 2008. Biochronology and biostratigraphy of the Upper Miocene of central Argentina: evidence from rodents and taphonomy. Geobios, 41:145-155. doi:10.1016/j.geobios.2006.09.005

Vucetich, M.G. \& Verzi, D.H. 1995. Los roedores Caviomorpha. In: M.T. Alberdi; G. Leone \& Tonni, E.P. (eds.) Evolución biológica y climática de la Región Pampeana durante los últimos cinco millones de años. Un ensayo de correlación con el Mediterráneo Occidental. Madrid, Museo de Ciencias Naturales de Madrid, Consejo de Investigaciones, p. 211-225 (Monografias 12).

Received in April, 2012; accepted in October, 2012. 